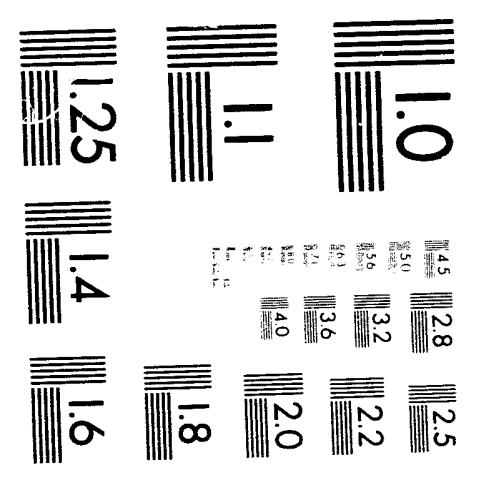



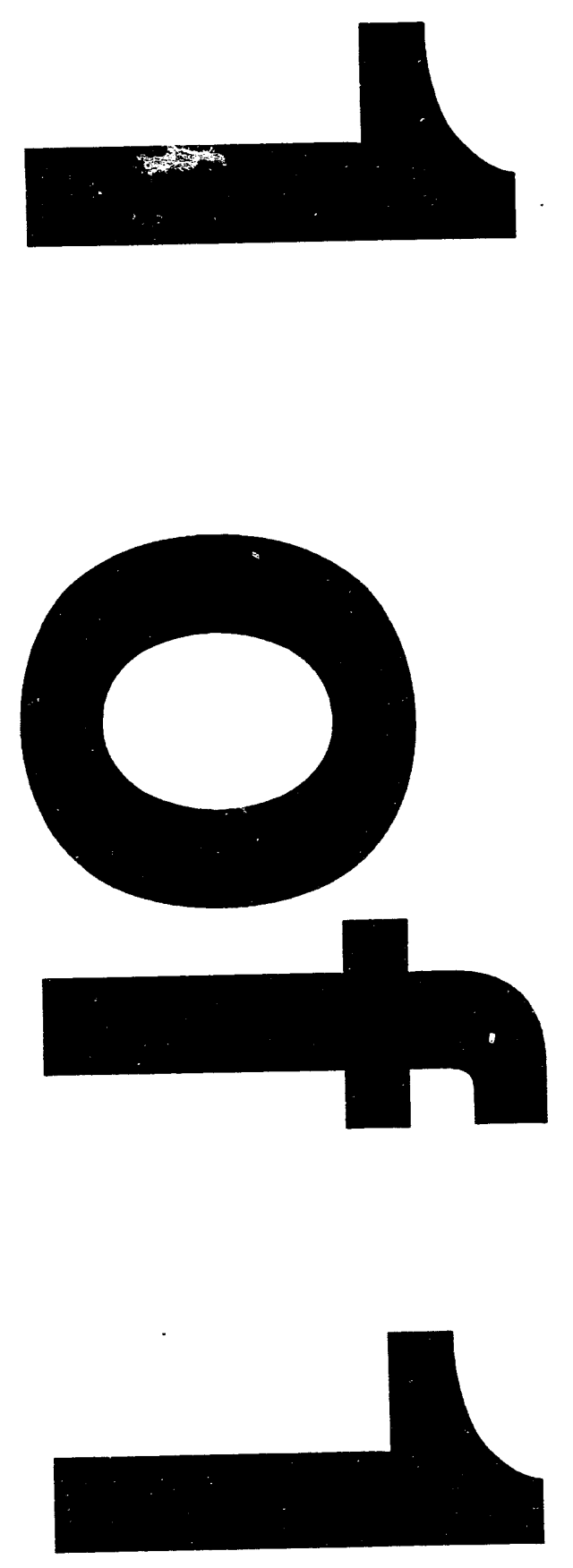


\section{INSTITUTE FOR FUSION STUDIES}

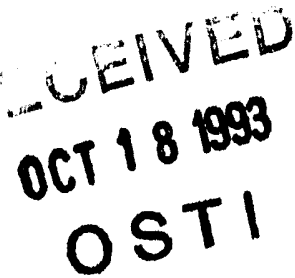

DOE/ET-53088-621

IFSR \#621

Continuum Damping of Ideal Toroidal

Alfvén Eigenmodes

X.D. Zhang, Y.Z. Zhang, ${ }^{a)}$ and S.M. MahajaN

Institute for Fusion Studies

The University of Texas at Austin

Austin, Texas 78712

August 1993

a) Permanent address: ICTP, Trieste, Italy

\section{THE UNIVERSITY OF TEXAS}

\section{AUSTIN}




\title{
Continuum Damping of Ideal Toroidal Alfvén Eigenmodes
}

\author{
X.D. Zhang, Y.Z. Zhang, ${ }^{a)}$ and S.M. Mahajan \\ Institute for Fusion Studies \\ The University of Texas at Austin \\ Austin, Texas 78712
}

\begin{abstract}
A perturbation theory based on the two dimensional (2D) ballooning transform is systematically developed for ideal toroidal Alfvén eigenmodes (TAEs). A formula, simIlar to the Fermi golden rule for decaying systems in quantum mechanics, is derived for the continuum damping rate of the TAE; the decay (damping) rate is expressed explicitly in terms of the coupling of the TAE to the continuum spectrum. Numerical results are compared with previous calculations. It is found that in some narrow intervals of the parameter $m \hat{\epsilon}$ the damping rate varies very rapidly. These regions correspond precisely to the root missing intervals of the numerical solution by Rosenbluth et al. [Phys. Fluids B 4, 2189 (1992)].
\end{abstract}

\section{DISCLAIMER}

\begin{abstract}
This report was prepared as an account of work sponsored by an agency of the United States Government. Neither the United States Government nor any agency thıreof, nor any of their employees, inakes any warranty, express or implied, or assumes any legal liability or responsibility for the accuracy, completeness, or usefulness of any information, apparatus, product, or process disclosed, or represents that its use would not infringe privately owned rights. Reference herein to any specific commercial product, process, or service by trade name, trademark, manufacturer, or otherwise does not necessarily constitute or imply its endorsement, recommendation, or favoring by the United States Government or any agency thereof. The views and opinions of authors expressed herein do not necessarily state or reflect those of the United States Government or any agency thereof.
\end{abstract}

Dermanent address: ICTP, Trieste, Italy 


\section{Introduction}

In recent years, much effort has been devoted to the study of shear Alfvén waves in a torus, in particular, to the study of toroidicity induced Alfvén eigenmodes (TAE). ${ }^{1-16}$ It was contended that the TAE's, localized within the toroidicity induced gaps of the shear Alfvén continuum, are more likely to be driven by external energy sources, because they are not damped by the phase mixing effect which greatly stablizes shear Alfven waves in the continuum. A proper understanding of the structure and stability of the TAE modes in a modern day tokamak is therefore of great importance, because these modes may limit the confinement time of fusion alpha particles with serious consequences for ignition in a reactor. Similar considerations would hold for experiments with high power neutral beams used for plasma heating or current drive.

Earlier theories ${ }^{4-6}$ considering the electron Landau damping through the curvature drifts as the only damping mechanism, showed a very low stability threshold for the TAE, while experiments performed on the TFTR ${ }^{7}$ and DIII- $\mathrm{D}^{8}$ suggested a much higher value. In order to give practical estimates of the stability threshold for the TAE, one must take into account all possible damping mechanisms present in the plasma. Recent theoretical studies revealed that other factors such as continuum resonance, ${ }^{9-12}$ trapped particle effects ${ }^{13}$ and finite parallel electric field effects ${ }^{14,16}$ (radiation damping) are indeed much more effective in damping the TAE. In a recent paper, ${ }^{15}$ it was also shown that the TAE's, instead of being true eigenmodes, were quasimodes associated with the analytical continuation of the dispersion function onto an unphysical sheet of its Riemann surface.

In a kinetic theory of shear Alfvén waves, both the radiation as well as continuum damping are manifestations of the same physical phenomenon: the interaction of the TAE with the kinetic Alfven wave (KAW). The former operates at the main defining ( $m$ number) gap of 
the mode while the latter is due to its coupling with the KAW's characterising the same $n$ but different $m$ numbers. The continuum damping, which can be comparable to the intrinsic radiation damping, is accessible only in a multiple gap theory.

In this paper, we will be concerned with the TAE continuum damping for moderate to high mode numbers $(n)$. The physical model is essentially the one used in Refs. 9 and 10. The motivation for doing yet another work on this problem comes from two diverse considerations. First, it is to provide a simple, physical, and unified way of calculating the continuum damping, a way which simultaneously reveals the quasimode nature of the TAE in a more transparent manner. In fact, the problem is analogous to a quantum mechanical decay or a transition problem: naturally the damping rate is nothing but the transition rate. The second purpose is to delineate a methodology for extending the ballooning approach for doing genuine $2 \mathrm{D}$ problems on a torus; this general, concise and reasonably rigorous formulation may be useful in solving other problems as well.

The paper is organized as follows: In Sec. II, we give a general guideline of the theoretical framework, where the continuum damping rate is derived in terms of the coupling between the discrete and continuum modes. Detailed calculations are carried out in Secs. III and IV. In Sec. III, the ballooning equation is solved approximately for the disctete as well as continuum modes near the first gap. The explicit eigenfunctions and coupling matrix elements are given in Sec. IV. In Sec. V, we give the numerical results, and compare them with previous calculations, and Sec. VI is devoted to brief summary and conclusions. 


\section{Perturbation Theory for Continuum Damping of the TAE}

We consider a circular, axisymmetric, large aspect ratio tokamak with a low beta plasma. The appropriate equation for the Alfven waves is ${ }^{9,10}$ :

$$
\begin{aligned}
\frac{d}{d x}\left[\frac{\Omega^{2} f(x)}{4}-(x-l)^{2}\right] \frac{d \phi_{l}}{d x} & -\frac{1}{s^{2}}\left[\frac{\Omega^{2} f(x)}{4}-(x-l)^{2}\right] \phi_{l} \\
+ & \frac{\epsilon \Omega^{2}}{4}\left(\frac{d^{2} \phi_{l+1}}{d x^{2}}+\frac{d^{2} \phi_{l-1}}{d x^{2}}\right)=0 .
\end{aligned}
$$

where $\phi_{l}$ is the poloidal Fourier component obtained from the physical electrostatic potential (with toroidal mode number $n$ ), $\Phi(x, \theta, \zeta) \equiv e^{i n \zeta-i m \theta} \sum_{l} e^{-i l \theta} \phi_{l}(x), \theta(\zeta)$ is the poloidal (toroidal) angle, $x \equiv n\left(q-q_{0}\right)$ is the radial variable, ( $q$ is the safety factor, $q_{0} \equiv \mathrm{m} / \mathrm{n}$ is the safety factor corresponding to the central poloidal Fourier component), $\Omega \equiv \omega / \omega_{A 0}$ is the mode frequency normalized to the Alfvén frequency $\omega_{A 0} \equiv\left[v_{A} /(2 R q)\right]_{q=q_{0}}, f(x) \equiv$ $\omega_{A 0}^{2} / \omega_{A}^{2}$ represents the radial variation of the Alfvén speed, $s \equiv(d \ln q / d \ln r)_{q=q 0}$ measures the magnetic shear, $\epsilon \equiv 5 r / 2 R$ is the toroidal coupling constant, and $R(r)$ is the major (minor) radius.

For toroidal Alfvén waves near the first gap, it is appropriate to consider $\Omega^{2} f(x) \equiv$ $1+\epsilon g(x)$ with $g(x) \approx g+2 x ; m \hat{\epsilon}+\ldots$, where $\hat{\epsilon} \equiv \epsilon /\left[\partial \ln \left(q^{2} / v_{A}^{2}\right) / \partial \ln q^{2}\right]_{q=q_{0}}$, and $g$ is the order unity eigenvalue measuring the frequency shift from the center of gap. Making use of the $2 \mathrm{D}$ ballooning transform ${ }^{17}$ (a detailed discussion of the transform is given in Appendix A),

$$
\phi_{l}(x)=\oint d \lambda d k \exp [i k(x-l)-i \lambda l] \hat{\varphi}(k, \lambda),
$$

and neglecting higher order effects, we obtain the mode equation in $k-\lambda$ space

$$
\left[\frac{\partial^{2}}{\partial k^{2}}+\frac{1+\epsilon g}{4}+\frac{\epsilon}{2} \cos (k+\lambda)-F(k)-\frac{i \epsilon}{2 m \hat{\epsilon}} \frac{\partial}{\partial \lambda}\right] \varphi(k, \lambda)=0
$$

where $\varphi \equiv \hat{\varphi} \sqrt{1+s^{2} k^{2}}$, and $F(k) \equiv s^{2} /\left(1+s^{2} k^{2}\right)^{2}$. 
In Eq. (1), the ballooning symmetry, i.e., the invariance under the translation $x \rightarrow$ $x+1, l \rightarrow l+1$, is broken by the radial dependence of $f(x)$. The appearance of the term proportional to $\partial / \partial \lambda$ in Eq. (3) is a manifestation of the broken symmetry. Notice that we have neglected higher order symmetry breaking effects [i.e. terms associated with $(x / m)^{2}$ or $\left.(l / m)^{2} \sim\left(1 / m^{2}\right) \partial^{2} / \partial \lambda^{2}\right]$. This is valid only if $(1 / m) \partial / \partial \lambda$ is a posteriori shown to be proportional to a small parameter intrinsic to ihe problem. In the case of TAE, fortunately, it turns out to be the case: the small parameter being $\epsilon$. Let us define the ballooning operator

$$
\mathcal{L}[\lambda] \equiv \frac{\partial^{2}}{\partial k^{2}}+\frac{\epsilon}{2} \cos (k+\lambda)-F(k)
$$

so that Eq. (3) can be rewritten as

$$
\left[\mathcal{L}[\lambda]+\frac{1+\epsilon g}{4}-\frac{i \epsilon}{2 m \bar{\epsilon}} \frac{\partial}{\partial \lambda}\right] \varphi(k, \lambda)=0
$$

and will be solved perturbatively. The Sturm-Liouville nature of $\mathcal{L}$ guarentees that its eigenfunctions form a complete set in $k$ space. The eigenfunctions are defined by

$$
\mathcal{L}[\lambda] \chi_{p}(k, \lambda)=-\frac{1+\epsilon \tilde{g}_{p}(\lambda)}{4} \chi_{p}(k, \lambda)
$$

with corresponding eigenvalues $\tilde{g}_{p}(\lambda)$. In Eq. (6) the $\lambda$ dependence of various quantities is merely parametric. We can now expand $\varphi(k, \lambda)$ in terms of the complete set $\chi_{p}$ :

$$
\varphi(k, \lambda)=\sum_{p} C_{p}(\lambda) \chi_{p}(k, \lambda)
$$

where the coefficients $C_{p}(\lambda)$ are projections of $\varphi(k, \lambda)$ onto the base functions $\chi_{p}(k, \lambda)$. Substituting Eq. (7) into Eq. (5), and carrying out the standard annihilation procedure (assuming the $\chi_{p}$ 's are appropriately normalized), we get

$$
\left(H_{p}-\frac{m \hat{\epsilon}}{2} g\right) C_{p}(\lambda)+\sum_{p^{\prime} \neq p} V_{p p^{\prime}} C_{p^{\prime}}(\lambda)=0,
$$

where

$$
H_{p} \equiv i \frac{d}{d \lambda}+\frac{m \hat{\epsilon}}{2} \tilde{g}_{p}(\lambda)+i\left\langle\chi_{p} \mid \frac{\partial \chi_{p}}{\partial \lambda}\right\rangle \text {, }
$$




$$
V_{p p^{\prime}} \equiv i\left\langle\chi_{p} \mid \frac{\partial \chi_{p^{\prime}}}{\partial \lambda}\right\rangle=i \int d k \chi_{p}^{*}(k, \lambda) \frac{\partial \chi_{p^{\prime}}(k, \lambda)}{\partial \lambda}
$$

and $\chi_{p}^{*}$ is the complex conjugate of $\chi_{p}$.

Before proceeding further, let us comment on the physical significance of the expansion procedure. It is well known that high-n ( $n$ is the toroidal number) modes in the toroidal plasma have a two length-scale structure: a fast scale for the mode centered at each rational surface, and a slow scale for the coupling between these modes through toroidicity. In the case of a weak violation of the ballooning symmetry, the first term in Eq. (8) is dominant. It is easy to confirm that the expansion coefficients $C_{p}(\lambda)$ in $\mathrm{Eq} .(7)$ describe the large scale envelop of the mode, while the fine structure of the mode is contained in the functions $\chi_{p}$ 's. The term with summation over $p^{\prime}$ is the coupling between modes of different frequencies. The conventional ballooning theory, ${ }^{19-22}$ while useful in various applications, can not accommodate these coupling terms. However, for the continuum damping of the TAE, it is exactly these terms that are relevant. For large $m \hat{\epsilon}$, it is appropriate to treat the coupling terms as a perturbation. The lowest order equations are then decoupled, and the eigenvalues are determined by requiring that $C_{p}(\lambda)$ be periodic in $\lambda$ :

$$
g_{p, N}^{(0)}=\frac{1}{2 \pi} \oint d \lambda \hat{g}_{p}(\lambda)-\frac{2 N}{m \hat{\epsilon}}
$$

where $N$ is an integer,

$$
\hat{g}_{p}(\lambda)=\tilde{g}_{p}(\lambda)+\frac{2 i}{m \hat{\epsilon}}\left\langle\chi_{p} \mid \frac{\partial \chi_{p}}{\partial \lambda}\right\rangle,
$$

with corresponding expansion coefficients (determining the eigenfunctions) given by

$$
C_{p, N}^{(0)}(\lambda)=\frac{1}{\sqrt{2 \pi}} \exp \left(-\frac{i m \hat{\varepsilon}}{2} \int_{0}^{\lambda} d \lambda^{\prime}\left[g_{p, N}^{(0)}-\hat{g}_{p}\left(\lambda^{\prime}\right)\right]\right) .
$$

Higher order corrections follow from the siandard perturbation theory used in quantum mechanics. We readily find that the first order correction to the eigenvalue $g_{p, N}^{(1)}=0$. The 
second order correction, given by

$$
g_{p, N}^{(2)}=\left(\frac{2}{m \hat{\epsilon}}\right)^{2} \sum_{N^{\prime}} \sum_{p^{\prime} \neq p} \frac{\left|\oint d \lambda C_{p, N}^{(0)}(\lambda) V_{p, p^{\prime}} C_{p^{\prime}, N^{\prime}}^{(0)}(\lambda)\right|^{2}}{g_{p, N}^{(0)}-g_{p^{\prime}, N^{\prime}}^{(0)}} .
$$

will be used in Sec. IV to calculate the continuum damping rate for the TAE.

\section{The Spectra of Ballooning Equation}

In Sec. II, we sketched the general methodology to be followed in this paper. In this section we explicitly solve the ballooning equation [Eq. (6)] for its eigenfunctions and eigenvalues. Because the coupling between the TAE and the continuum modes is strongest near the gap edges, it will suffice to calculate solutions pertinent to the range $|\tilde{g}| \sim 1$. Noticing that the term $F$ is localised in a region $|k| \sim 1 / s$, we divide the $k$ space into three regions. (I): $k<-1 / s$, (II): $|k| \leq 1 / s$, (III): $k>1 / s$. In regions (I) and (III) the $F$ term can be ignored, while in region (II), the toroidal coupling term can be ignored. The spectrum of Eq. (6) becomes clear from a consideration of solutions in the regions (I) and (III). In these regions, it reduces to a Matthew equation whose spectrum is known to be divided into bands of 'stable' and 'unstable' solutions, corresponding respectively to the continuum and gaps in the shear Alfvén spectra. In these outer regions, for Alfvén waves near the first gap, it is appropriate to write $\chi(k, \lambda)$ as ${ }^{1,16,23}$

$$
\chi(k, \lambda)=\chi_{+} e^{-i k / 2}+\chi_{-} e^{i k / 2}
$$

where the exponential parts carry the fast variaiion (of order unity), and $\chi_{+}$and $\chi_{-}$are

relatively slow varying. Substitute Eq. (15) into Eq. (6) and averaging out the fast variations, we obtain the set

$$
\begin{aligned}
& \chi_{+}^{\prime \prime}-i \chi_{+}^{\prime}+\frac{\epsilon}{4} \tilde{g} \chi_{+}+\frac{\epsilon}{4} e^{-i \lambda} \chi_{-}=0 \\
& \chi_{-}^{\prime \prime}+i \chi_{-}^{\prime}+\frac{\epsilon}{4} \tilde{g} \chi_{-}+\frac{\epsilon}{4} e^{i \lambda} \chi_{+}=0 .
\end{aligned}
$$


It is apparent that $\chi_{ \pm}^{\prime} \sim \mathcal{O}(\epsilon)$, and the second order derivatives in Eqs. (16) and (17) can be neglected. With this simplification, and elimination of $\chi_{-}$from Eqs. (16) and (17) we obtain

$$
\chi_{+}^{\prime \prime}=-\left(\frac{\epsilon}{4}\right)^{2}\left(\tilde{g}^{2}-1\right) \chi_{+}
$$

clearly revealing that the gap edges are defined by $\tilde{g}= \pm 1$. For $|\tilde{g}|<1$, the solution is 'unstable,' while for $|\tilde{g}|>1$ the solution is 'stable.'

The behavior of $\chi(k, \lambda)$ is considerably more complex in region (II). In general, we can not solve it analytically. But for moderate to large shear, the region (II) is narrow, and for practical purposes we don't need the explicit form of $\chi(k, \lambda)$ in this region. The eigenmodes can be approximately obtained by solving Eqs. (16) and (17) in the outer regions and matching the solutions through region (II)..( A similar method was used in Ref. 23). And the inner product (used to compute quantities of interest) can be conveniently calculated from the outer regions alone,

$$
\langle f \mid g\rangle=\left(\int_{-\infty}^{-1 / \bullet} d k+\int_{1 /,}^{\infty} d k\right) f^{*}(k, \lambda) g(k, \lambda)
$$

because the contribution from region (II) is of order $\epsilon$ of the contributions from the outer regions.

\section{A. Matching rules}

In region (II), the $F$ term is dominant, and the toroidal coupling term in Eq. (6) can be neglected. Because the characteristic large scale lengths of $\chi_{+}$and $\chi_{-}$, their variations can not be seen by $F$ (i.e. in the region where $F$ is finite the variation of $\chi_{ \pm}$is negligible). Thus $\chi[E q .(15)]$ appears as a plane wave, and the effects of the $F$ term can be approximately calculated by studying the scattering of a plane wave with wavenumber $\tilde{k}=1 / 2$ in the presence of a potential $F(k)$. Since $F$ is symmetric, its eigenfunctions must have either even or odd parity. In regions (I) and (III), these functions, aside from an arbitrary overall 
numerical factor, can be written in the following form :

$$
\begin{aligned}
& \chi_{0}= \begin{cases}\sin \left(k / 2-\delta_{1}\right) ; & \text { in region (I) } \\
\sin \left(k / 2+\delta_{1}\right) ; & \text { in region (III) }\end{cases} \\
& \chi_{e}= \begin{cases}\cos \left(k / 2-\delta_{2}\right) ; & \text { in region (I) } \\
\cos \left(k / 2+\delta_{2}\right) ; & \text { in region (III) }\end{cases}
\end{aligned}
$$

where $\chi_{o}\left(\chi_{e}\right)$ has odd (even) parity. These eigenfunctions, and as a result the numerical values of $\delta_{1}$ and $\delta_{2}$, are easily obtained with a shooting code. Table I gives the values of $\delta_{1}$ and $\delta_{2}$ for various values of the shear parameter $s$.

Near the edges of region (II), the slowly varying functions $\chi_{ \pm}$can be replaced by constants. The general form of the eigenfunction, with $F(k)$ in (II) inducing a phase shift, can be written as:

$$
\chi(k, \lambda)= \begin{cases}e^{-i k / 2}+C_{1} e^{i k / 2} & \text { in region (I) } \\ \tau\left(C_{r} e^{-i k / 2}+e^{i k / 2}\right) & \text { in region (III) }\end{cases}
$$

up to an overall arbitrary numerical factor. The matching rules relate $C_{r}$ and $\tau$ to $C_{l}$. By writing $\chi$ as the superposition of $\chi_{e}$, and $\chi_{o}$, we get the following expressions for $C_{r}$ and $\tau$ :

$$
\begin{gathered}
C_{r}=\frac{\left(1-D_{1} D_{2}\right)-i D_{2}\left(1+C_{l}\right)+i D_{1}\left(C_{1}-1\right)}{C_{l}\left(1-D_{1} D_{2}\right)+i D_{2}\left(1+C_{l}\right)+i D_{1}\left(C_{l}-1\right)} \\
\tau=\frac{\left(1-D_{1} D_{2}+i D_{1}+i D_{2}\right) C_{1}+i\left(D_{2}-D_{1}\right)}{1+D_{1} D_{2}}
\end{gathered}
$$

where $D_{1}=\tan \delta_{1}, D_{2}=\tan \delta_{2}$.

\section{B. Discrete mode}

The discrete mode(s) $(|\tilde{g}|<1)$, charaterized by evanescent solutions in both regions (I) and (III), can be represented as

$$
x_{+}= \begin{cases}-\tau e^{-i \lambda}\left[\tilde{g}-i \sqrt{1-\tilde{g}^{2}}\right] e^{-\alpha_{g} k} & \text { in region (III) } \\ e^{\alpha_{g} k} & \text { in region (I) }\end{cases}
$$


where $\alpha_{g}=\epsilon / 4 \sqrt{1-\tilde{g}^{2}}$. Substituting this back into Eq. (16), we obtain:

$$
\chi_{-}=\left\{\begin{array}{ll}
\tau e^{-\alpha_{0} k} & \text { in region (III) } \\
e^{i \lambda}\left[i \sqrt{1-\tilde{g}^{2}}-\tilde{g}\right] e^{a_{0} k} & \text { in region (I) }
\end{array},\right.
$$

and consequently

$$
\begin{aligned}
& C_{l}=e^{i \lambda}\left(i \sqrt{1-\tilde{g}^{2}}-\tilde{g}\right), \\
& C_{r}=\frac{e^{-i \lambda}}{-i \sqrt{1-\tilde{g}^{2}}-\tilde{g}} .
\end{aligned}
$$

Substituting them into the matching rule [Eq. (21)], we get the following equation for $\tilde{g}$ as a function of $\lambda$ :

$$
-\frac{1-D_{1} D_{2}}{D_{2}-D_{1}} \sqrt{1-\tilde{g}^{2}}+\frac{D_{1}+D_{2}}{D_{2}-D_{1}} \tilde{g}=\cos \lambda \text {. }
$$

Without loss of generality, we choose the $k$ space mode number $p=0$ for the discrete mode, and use $g_{0}(\lambda)$ to denote the solution to Eq. (27). Because $\left|g_{0}(\lambda)\right|<1$, we can write $g_{0}(\lambda)$ as:

$$
g_{0}(\lambda)=\cos [\Theta(\lambda)]
$$

which serves as the definition of $\Theta$ as a function of $\lambda$. Apparently, we can choose $\theta \in[0, \pi)$. Using the expression for $C_{l}[\mathrm{Eq} .(25)]$ in Eq. (22), we could write

$$
\tau(\lambda)=-\tau_{R} e^{i \lambda}
$$

where

$$
\tau_{R}(\lambda)=\frac{\left(1-D_{1} D_{2}\right) \cos \theta+\left(D_{1}+D_{2}\right) \sin \theta+\left(D_{1}-D_{2}\right) \sin \lambda}{1+D_{1} D_{2}},
$$

with $\sin \theta=\sqrt{1-g_{0}^{2}}$. Thus the normalized eigenfunction for the discrete mode can be written as:

$$
\chi_{0}(k, \lambda)=\left(\chi_{+} e^{-i k / 2}+\chi_{-} e^{i k / 2}\right) / N_{0}
$$


where $\chi_{+}$and $\chi_{-}$are given in Eqs. (23) and (24) and

$$
N_{0}=\left(\frac{4}{\epsilon} \frac{1+\tau_{R}^{2}}{\sqrt{1-g_{0}^{2}}}\right)^{1 / 2}
$$

is the normalization factor.

\section{Continuum modes}

The continuum modes $(|\tilde{s}|>1)$, are oscillatory for large $|k|$, and can be described by

$$
\chi_{+}=e^{i \alpha k}+B e^{-i \alpha k} \quad \text { in regions (I) and (III) }
$$

where $\alpha \equiv(\epsilon / 4) \sqrt{\tilde{g}^{2}-1}$, and $B$ is an arbitrary constant. Thus the most general form of the eigenfunction in the outer regions can be written as:

$$
\chi(k, \lambda)=\left(e^{i \alpha k}+B e^{-i \alpha k}\right) e^{-i k / 2}+\left[-(\beta+\tilde{g}) e^{i \alpha k}+B(\beta-\tilde{g}) e^{-i \alpha k}\right] e^{i \lambda+i k / 2}
$$

in region (I), and

$$
\chi(k, \lambda)=\tau\left\{\left(e^{-i \alpha k}+\tilde{B} e^{i \alpha k}\right) e^{i k / 2}+\left[-(\beta+\tilde{g}) e^{-i \alpha k}+\tilde{B}(\beta-\tilde{g}) e^{i \alpha k}\right]\right\} e^{-i \lambda-i k / 2}
$$

in region (III), where $\beta \equiv \sqrt{\tilde{g}^{2}-1}, \quad \alpha \equiv(\epsilon / 4) \beta$, and $\tau, B$ and $\widetilde{B}$ are constants independent of $k$.

The matching rules again determine $\widetilde{B}$ and $\tau$ in terms of $B$. In general, the continuum eigenfunctions are doubly degenerate, because we have one free parameter $B$. For definiteness, we choose $\tilde{B}=B$, and use the matching rules to solve $B$ and $\tau$ as functions of $\lambda$

$$
\begin{aligned}
& C_{l}=\frac{[-(\beta+\tilde{g})+B(\beta-\tilde{g})] e^{i \lambda}}{1+B} \\
& C_{r}=\frac{[-(\beta+\tilde{g})+B(\beta-\tilde{g})] e^{-i \lambda}}{1+B} .
\end{aligned}
$$


Substituting these into Eqs. (21) and (22), we find:

$$
B^{ \pm}=\frac{2 \beta}{\beta-\tilde{g}-Y^{ \pm}}-1
$$

where

$$
Y^{ \pm}=\frac{-i\left(D_{2}-D_{1}\right) \cos \lambda \pm \sqrt{\left(1+D_{1} D_{2}\right)^{2}+\left(D_{2}-D_{1}\right)^{2} \sin ^{2} \lambda}}{\left(1-D_{1} D_{2}\right)+i\left(D_{1}+D_{2}\right)}
$$

and

$$
\begin{aligned}
\tau^{ \pm} & =\frac{\left(1-D_{1} D_{2}+i D_{1}+i D_{2}\right) Y e^{i \lambda}+i\left(D_{2}-D_{1}\right)}{1+D_{1} D_{:}} \\
& =\tau_{\tau}^{ \pm} e^{i \lambda}
\end{aligned}
$$

with

$$
\tau_{r}^{ \pm}=\frac{\left(D_{2}-D_{1}\right) \sin \lambda \pm \sqrt{\left(1+D_{1} D_{2}\right)^{2}+\left(D_{2}-D_{1}\right)^{2} \sin ^{2} \lambda}}{1+D_{1} D_{2}}
$$

The plus and minus signs give the two linearly independent solutions for the same value of $\tilde{g}$. Noticing that $\tau_{r}^{+} \tau_{r}^{-}=-1$, it is straight-forward to verify that these two functions are orthogonal to each other (with Eq. (19) as the definition for the inner product).

Noticing that $Y^{ \pm}$is a unit vector in the complex plane, we can rewrite $B^{ \pm}$as:

$$
B^{ \pm}=(\beta+\tilde{g}) e^{i \theta_{ \pm}}
$$

which in turn defines the functions $\Theta_{ \pm}$. The continuum eigenfunctions can now be written in a more convenient way: in terms of the functions $\Theta_{ \pm}$. The normalized eigenfunctions for the continuum modes are:

$$
\chi_{\tilde{g}}^{(+)}(k, \lambda)=\left\{\begin{array}{c}
\left(1 / N_{c}\right)\left\{\left[e^{i \alpha k}+(\beta+\tilde{g}) e^{i \theta_{+}-i \alpha k}\right] e^{-i k / 2}\right. \\
\left.+\left[-(\beta+\tilde{g}) e^{i \alpha k}-e^{i \theta_{+}-i \alpha k}\right] e^{i \lambda+i k / 2}\right\} \text { in region (I) } \\
\left(\tau_{r} / N_{c}\right)\left\{\left[e^{-i \alpha k}+(\beta+\tilde{g}) e^{i \theta_{+}+i \alpha k}\right] e^{i k / 2+i \lambda}\right. \\
\left.+\left[-(\beta+\tilde{g}) e^{-i \alpha k}-e^{i \theta_{+}+i \alpha k}\right] e^{-i k / 2}\right\} \quad \text { in region (III) }
\end{array}\right.
$$




$$
\chi_{\tilde{g}}^{(-)}(k, \lambda)=\left\{\begin{array}{c}
-\left(\tau_{r} / N_{c}\right)\left\{\left[e^{i \alpha k}+(\beta+\tilde{g}) e^{i \theta_{-}-i \alpha k}\right] e^{-i k / 2}\right. \\
\left.+\left[-(\beta+\tilde{g}) e^{i \alpha k}-e^{i \theta_{-}-i \alpha k}\right] e^{i \lambda+i k / 2}\right\} \quad \text { in region (I) } \\
\left(1 / N_{c}\right)\left\{\left[e^{-i \alpha k}+(\beta+\tilde{g}) e^{i \Theta_{-}+i \alpha k}\right] e^{i k / 2+i \lambda}\right. \\
\left.+\left[-(\beta+\tilde{g}) e^{-i \alpha k}-e^{i \Theta_{-}+i \alpha k}\right] e^{-i k / 2}\right\} \quad \text { in region (III) }
\end{array}\right.
$$

where the normalization constant $N_{c}=\sqrt{4 \pi\left(1+\tau_{r}^{2}\right) \tilde{g}(\beta+\tilde{g})}$. These eigenfunctions are $\delta$. function normalized with respect to the variable $\alpha=\epsilon / 4 \sqrt{\tilde{g}^{2}-1}$. For the calculation of the coupling matrix elements, we shall use a more convenient set of base functions $\tilde{\chi}_{\tilde{g}}^{( \pm)}$:

$$
\tilde{\chi}_{\tilde{g}}^{( \pm)}=i e^{-i \theta_{ \pm} / 2} \chi_{\tilde{g}}^{( \pm)}
$$

It's easy to see that $\tilde{\chi}_{\tilde{g}}^{( \pm)}$equals a real normalized function times a phase factor $e^{i \lambda / 2}$. This property greatly simplifies the calculations.

\section{Calculations in the Second Dimension}

In order to use the perturbation theory expressions in Sec. II, we need to calculate the coupling matrix elements. For the self-coupling of the discrete mode, this is straight forward. The discrete eigenfunction given by Eq. (31) can be put in the form

$$
\chi_{0}(k, \lambda)=-i e^{-i(\theta-\lambda) / 2} \tilde{\chi}_{0}(k, \lambda)
$$

where $\tilde{\chi}_{0}$ is a real and normalized function. Thus the self-coupling for the discrete mode is

$$
\begin{aligned}
\left\langle\chi_{0} \mid \frac{\partial \chi_{0}}{\partial \lambda}\right\rangle & =\left\langle\tilde{\chi}_{0} \mid \frac{\partial \tilde{\chi}_{0}}{\partial \lambda}\right\rangle-\frac{i}{2} \frac{\partial(\Theta-\lambda)}{\partial \lambda} \\
& =-\frac{i}{2}\left(\frac{\partial \Theta}{\partial \lambda}-1\right),
\end{aligned}
$$

which, when substituted into Eqs. (11) and (13), yields

$$
g_{0 . N}^{(0)}=-\frac{2}{m \hat{\epsilon}}\left(N+\frac{1}{2}\right)+\overline{\cos \theta}
$$


and

$$
\begin{aligned}
C_{0, N}^{(0)}(\lambda) & =\frac{1}{\sqrt{2 \pi}} \exp \left(\frac{i \Theta}{2}+i N \lambda+i \frac{m \hat{\epsilon}}{2} \int_{0}^{\lambda} d \lambda^{\prime}\left(\cos \Theta\left(\lambda^{\prime}\right)-\overline{\cos \Theta}\right)\right) \\
& =e^{i N \lambda} C_{0,0}^{(0)}(\lambda)
\end{aligned}
$$

with

$$
\overline{\cos \Theta}=\frac{1}{\pi \pi} \oint \cos \Theta d \lambda
$$

The interesting point here is that although we did the Fourier expansion assuming the TAE mode is centered about a rational surface, the quantization rule [Eq. (47)] carries the mode to a place in between two rational surfaces - the place where the TAE should be located. This is seen most clearly in the small shear limit, where the function $\Theta(\lambda)$ approaches a constant, the mode resides half way in between the two mode rational surfaces, giving the usual single gap structure. The corresponding real space mode structure for the TAE is:

$$
\begin{aligned}
\Phi_{0, N}^{(0)}(x, \theta) & =\oint d \lambda C_{0, N}^{(0)}(\lambda) \sum_{l} e^{-i l(\theta+\lambda)} \int d k e^{i k(x-l)} \frac{\chi_{0}(k, \lambda)}{\sqrt{1+s^{2} k^{2}}} \\
& =e^{-i N \theta} \oint d \lambda C_{0, N}^{(0)}(\lambda) \sum_{l} e^{-i l(\theta+\lambda)} \int d k e^{i k(x-1-N)} \frac{\chi_{0}(k, \lambda)}{\sqrt{1+s^{2} k^{2}}} \\
& =e^{-i N \theta} \Phi_{0,0}^{(0)}(x-N, \theta) .
\end{aligned}
$$

Thus the significance of the quantum number $N$ is to shift the $N=0$ mode by $N$ rational surfaces, which is equivalent to do the mode expansion at another rational surface with $m^{\prime}=m+N$ and take the $N=0$ mode there. Of course this is a consequence of the symmetry properties peculiar to our present model, i.e. a consequence of the linear profile assumption for the local Alfvén speed. One point of caution in practice is that $N$ can not be too large lest the mode is carried out of the region of validity of the origional assumptions.

The calculations for the continuum modes in the second dimension is a little bit more complicated. Because these modes are degenerate, any ortho-normal set of functions, $\tilde{\chi}_{\tilde{g}}^{( \pm)}$as 
well as any linear combination of them can be used as the base functions. This causes some uncertainty in the cross coupling terms. For definiteness, we need to include the coupling term between $\tilde{\chi}_{\tilde{g}}^{(+)}$and $\tilde{\chi}_{\tilde{g}}^{(-)}$in the lowest order continuum equations. Thus we have

$$
\begin{aligned}
& {\left[\frac{d}{d \lambda}+\left\langle\tilde{\chi}_{\tilde{g}}^{(+)} \mid \frac{\partial \tilde{\chi}_{\tilde{g}}^{(+)}}{\partial \lambda}\right\rangle+i \frac{m \hat{\epsilon}}{2}(g-\tilde{g})\right] C_{(+)}(\lambda)=-\left\langle\tilde{\chi}_{\tilde{g}}^{(+)} \mid \frac{\partial \tilde{\chi}_{\tilde{g}}^{(-)}}{\partial \lambda}\right\rangle C_{(-)}(\lambda)} \\
& {\left[\frac{d}{d \lambda}+\left\langle\tilde{\chi}_{\tilde{g}}^{(-)} \mid \frac{\partial \tilde{\chi}_{\tilde{g}}^{(-)}}{\partial \lambda}\right\rangle+i \frac{m \hat{\epsilon}}{2}(g-\tilde{g})\right] C_{(-)}(\lambda)=-\left\langle\tilde{\chi}_{\tilde{g}}^{(-)} \mid \frac{\partial \tilde{\chi}_{\tilde{g}}^{(+)}}{\partial \lambda}\right\rangle C_{(+)}(\lambda)}
\end{aligned}
$$

as the zeroth order equation for the continuum modes.

To get around the complexity of solving Eqs. (53) and (54) simultaneously, we look for a unitary transfomations from $\tilde{\chi}_{\tilde{g}}^{( \pm)}$to a new set $\psi_{\tilde{g}}^{( \pm)}$such that the cross coupling term in the new representation is zero, i.e.

$$
\left\langle\psi_{g}^{(+)} \mid \frac{\partial \psi_{g}^{(-)}}{\partial \lambda}\right\rangle=\left\langle\psi_{\vec{g}}^{(-)} \mid \frac{\partial \psi_{g}^{(+)}}{\partial \lambda}\right\rangle=0 .
$$

Without loss of generality, we can write

$$
\begin{aligned}
& \psi_{\tilde{g}}^{(+)}=e^{i \xi_{1}} \sin \gamma \tilde{\chi}_{\tilde{g}}^{(+)}+e^{i \xi_{2}} \cos \gamma \tilde{\chi}_{\tilde{g}}^{(-)} \\
& \psi_{\tilde{g}}^{(-)}=e^{i \xi_{3}} \cos \gamma \tilde{\chi}_{\tilde{g}}^{(+)}+e^{i \xi_{1}} \sin \gamma \tilde{\chi}_{\tilde{g}}^{(-)},
\end{aligned}
$$

where $\gamma \in[0, \pi / 2],\left(\xi_{1}-\xi_{2}\right)-\left(\xi_{3}-\xi_{4}\right)=\pi, \gamma$ and $\xi_{i}$ 's are arbitrary periodic functions of $\lambda$. Substituting Eq. (56) into Eq. (55) yields

$$
\begin{aligned}
\frac{d z}{d \lambda}= & \left(\left\langle\tilde{\chi}_{\tilde{g}}^{(+)} \mid \frac{\partial \tilde{\chi}_{\tilde{g}}^{(+)}}{\partial \lambda}\right\rangle-\left\langle\tilde{\chi}_{\tilde{g}}^{(-)} \mid \frac{\partial \tilde{\chi}_{\tilde{g}}^{(-)}}{\partial \lambda}\right\rangle\right) z \\
& -z^{2}\left\langle\tilde{\chi}_{\tilde{g}}^{+} \mid \frac{\partial \tilde{\chi}_{\tilde{g}}^{-}}{\partial \lambda}\right\rangle-\left\langle\tilde{\chi}_{\tilde{g}}^{+} \mid \frac{\partial \tilde{\chi}_{\tilde{g}}^{-}}{\partial \lambda}\right\rangle^{*}
\end{aligned}
$$

where $z=e^{-i\left(\xi_{1}-\xi_{2}\right)} \tan \gamma$. Substituting $\tilde{\chi}_{\tilde{g}}^{( \pm)}$given by Eq. (43) into the above equation, we find that the first term is zero, the other two terms are real. Therefore, $z= \pm i$ are the 
exact solutions of Eq. (57). Without loss of generality, we choose $z=i$. The new set of base functions are

$$
\psi_{\tilde{g}}^{( \pm)}(k, \lambda)=\frac{1}{\sqrt{2}}\left(\tilde{\chi}_{\tilde{g}}^{(+)} \mp i \tilde{\chi}_{\tilde{g}}^{(-)}\right)
$$

In the new basis, it is straightforward to calculate the self-coupling matrix elements:

$$
\begin{aligned}
& \left\langle\psi_{g}^{(+)} \mid \frac{\partial \psi_{\tilde{g}}^{(+)}}{\partial \lambda}\right\rangle=\frac{i}{2}+i\left\langle\tilde{\chi}_{\tilde{g}}^{(-)} \mid \frac{\partial \tilde{\chi}_{\tilde{g}}^{(+)}}{\partial \lambda}\right\rangle \\
& \left\langle\psi_{\tilde{g}}^{(-)} \frac{\partial \psi_{\tilde{g}}^{(-)}}{\partial \lambda}\right\rangle=\frac{i}{2}-i\left\langle\tilde{\chi}_{\tilde{g}}^{(-)} \mid \frac{\partial \tilde{\chi}_{\tilde{g}}^{(+)}}{\partial \lambda}\right\rangle,
\end{aligned}
$$

where

$$
\begin{gathered}
\left\langle\mid \frac{\partial \tilde{\chi}_{\tilde{g}}^{(+)}}{\partial \lambda}\right\rangle=S(\lambda) \delta\left(\alpha-\alpha^{\prime}\right), \\
S(\lambda)=\tau_{r} \frac{\frac{\left(D_{2}-D_{1}\right) \cos \lambda}{\sqrt{\left(1+D_{1} D_{2}\right)^{2}+(D} \cdot \frac{\left.D_{1}\right)^{2} \sin ^{2} \lambda}{g} \cos \left(\frac{\Theta_{+}-\Theta_{-}}{2}\right)-\beta \sin \left(\frac{\Theta_{+}-\Theta_{-}}{2}\right)}}{\left(1+\tau_{r}^{2}\right) \tilde{g}},
\end{gathered}
$$

and $\alpha \equiv \epsilon / 4 \sqrt{\tilde{g}^{2}-1}$. Substituting these into Eqs. (11) and (13) for the zeroth order eigenvalue and eigenfunction, we get

$$
\begin{aligned}
g_{\tilde{g}, N}^{(0) \pm} & =\tilde{g}-\frac{2 N+1}{m \hat{\epsilon}} \mp \frac{2}{m \hat{\epsilon}} \overline{S(\lambda)} \\
C_{\tilde{g}, N}^{(0) \pm}(\lambda) & =\frac{1}{\sqrt{2 \pi}} \exp \left[i N \lambda \mp i \int_{0}^{\lambda} d \lambda^{\prime}\left(\overline{S(\lambda)}-S\left(\lambda^{\prime}\right)\right)\right]
\end{aligned}
$$

where

$$
\overline{S(\lambda)}=\frac{1}{2 \pi} \oint d \lambda S(\lambda)
$$

Equation (14) can now be used to calculate the continuurn damping. We need only consider the $N=0$ case. Because the spectrum is a continuum except for the discrete mode, the summation over $p^{\prime}$ should be replaced with an integral

$$
g_{0,0}^{(2)}=\left(\frac{2}{m \tilde{\epsilon}}\right)^{2} \sum_{N} \int d \tilde{g} \frac{\left|\oint d \lambda C_{\tilde{g}, N}^{*(0)}(\lambda) V_{0, \tilde{g}}(\lambda) C_{0,0}^{(0)}(\lambda)\right|^{2}}{g_{0,0}^{(0)}-g_{\tilde{g}, N}^{(0)}},
$$


where $|\tilde{g}|>1$ and the ballooning eigenfunctions are $\delta$-function normalized with respect to $\tilde{g}$. For the eigenfunctions given by Eq. (43), where they are $\delta$-function normalized with respect to the variable $\alpha=(\epsilon / 4) \sqrt{\tilde{g}^{2}-}$, Eq. (66) should be changed to

$$
g_{0,0}^{(2)}=\left(\frac{2}{m \tilde{\epsilon}}\right)^{2} \sum_{N} \int d \tilde{g} \frac{\epsilon}{4} \frac{\tilde{g}}{\sqrt{\tilde{g}^{2}-1}} \frac{\left|\oint d \lambda C_{\tilde{g}, N}^{*(0)}(\lambda) V_{0, \tilde{g}}(\lambda) C_{0,0}^{(0)}(\lambda)\right|^{2}}{g_{0,0}^{(0)}-g_{\tilde{g}, N}^{(0)}} .
$$

Notice that the integrand in Eq. (67) has a first order singularity at the resonance points $g_{\tilde{g}, N}^{(0)}=g_{0,0}^{(0)}$. The contour of the integral should be deformed as in the Landau-damping problem. If positive real frequency is adpoted, the correct contour is determined by giving the variable $g_{0,0}^{(0)}$ a small positive imaginary part. Thus

$$
\operatorname{Im}\left[g_{0,0}^{(2)}\right]=-\frac{4 \pi i}{(m \hat{\epsilon})^{2}} \frac{\epsilon}{4} \sum_{N} \frac{\tilde{g}_{N}}{\sqrt{\tilde{g}_{N}^{2}-1}}\left|\oint d \lambda C_{\tilde{g}_{N}, N}^{*(0)}(\lambda) V_{0, \tilde{g}_{N}}(\lambda) C_{0,0}^{(0)}(\lambda)\right|^{2}
$$

where $\tilde{g}_{N}$ is the root of $g_{\tilde{g}, N}^{(0)}=g_{0,0}^{(0)}$.

The explicit forms of the coupling matrix elements between the discrete and continuum modes are given in the Appendix B.

\section{Numerical Results}

The continuum dainping rates for various values of the shear parameter (s) are obtained by numerically evaluating the right hand side of Eq. (68). Results for $s=0.5,1.0,1.5,2.0$ are shown in Fig. 1. In all cases, the damping rate changes rapidly with the variation of the parameter $m \hat{\epsilon}$, which specifies the slope of linear profile for the local Alfven speed. This is because the coupling between the TAE and the continuum modes is concentrated near the gap edges, i.e. the maximum absolute value of $V_{0, \tilde{g}}(\lambda)$ appears at some place $|\tilde{g}| \sim 1+\varepsilon$. When $m \hat{\epsilon}$ is changed, it moves the TAE in and out of this region of maximum coupling, causing the dampig rate to go higher and lower. On the other hand, when $s$ becomes larger, this area of concentration becomes narrower, thus the damping rate oscillates more violently with $m \hat{\epsilon}$. It is readily observable that the continuum damping rate grows with larger shear. 
This increase comes from two factors: first, the radial extent of the TAE increases with shear, causing more resonance points to come into the effective range of the TAE; second, the maximum value of the coupling matrix element $V_{0, \tilde{g}}(\lambda)$ also increases with shear.

Figure 2 compares our present calculations with that of Ref. 11 for the case $s=0.8$. The results are in good agreement, except in the latter there are regions in $m \hat{\epsilon}$ where solutions could not be found. It is clear that our approach yields the damping as a continuous function of $m \hat{\epsilon}$; there are no missing intervals where the roots could not be found. The regions [in $m \tilde{\epsilon}]$ of rapid variation and turning around of the damping rate coincide with the intervals which could not be handled by the methods of Ref. 11 .

\section{Summary and Conclusions}

The continuum damping effects on the toroidal Alfvén eigenmodes are calculated by using a ballooning approach. The origional $2 \mathrm{D}$ eigenvalue problem is converted into an eigenvalue problem in the usual ballooning space and a set of first order differential equations for the global structure and eigenvalue in a second dimension. The ballooning space ( $k$-space) eigenfunctions are solved semi-analytically, while solutions in the second dimension ( $\lambda$-space) are obtained by the standard perturbation theory. The continuum damping rate is written as a summation of contributions from the resonance points, each term resembling the Fermi golden rule formula. This clearly shows the quasi-mode nature of the continuum damped $T A E$, and indicates that the continuum damping can be properly interpreted as a transition process, i.e. the decaying of the TAE mode into the continuum modes. Numerical results are obtained for various shear parameters, which is in agreement with earlier calculations. The analysis also fills in the gaps left by earlier calculations; there are no intervals in the parameter $m \hat{\epsilon}$ where the roots could not be found. The damping rate is oscillatory and not a monotonic decreasing function of $m \hat{\epsilon}$. Although there is an overall decrease, the oscillatory behavior is dominant, especially for strong shear and moderate mode numbers. 


\section{Appendix A: Comments on the Ballooning Transform}

At the heart of our presen approach is the 2D ballooning transform Eq. (2) and the expansion Eq. (7). The validity of the approach is based on the fact that the system approximately obeys the so called ballooning invariance. Although the form used in the text is simpler and more straight forward in carrying out the calculations, a better understanging can be gained by an investigation in the real space. The equation under consideration is

$$
\begin{aligned}
\frac{d}{d x}\left[\mu-(x-l)^{2}\right] \frac{d \phi_{l}}{d x} & -\frac{1}{s^{2}}\left[\mu-(x-l)^{2}\right] \phi_{l} \\
+ & \frac{\epsilon \Omega^{2}}{4}\left(\frac{d^{2} \phi_{l+1}}{d x^{2}}+\frac{d^{2} \phi_{l-1}}{d x^{2}}\right)=0,
\end{aligned}
$$

which is the same as Eq. (1), except that $f(x)$ is replaced with a constant. Eq. (A1) is invariant under the transformation $x \rightarrow x+1 ; l \rightarrow l+1$. Thus we may assume

$$
\phi_{1}(x)=e^{-i l \lambda} F(x-l)
$$

with an arbitrary phase factor $\lambda \in[0,2 \pi]$. Substituting Eq. (A2) ints Eq. (A1) we get

$$
\mathcal{H}(k, \lambda) \chi(k, \lambda)=-\mu \chi(k, \lambda)
$$

where

$$
\begin{gathered}
\mathcal{H}(k, \lambda)=\frac{d^{2}}{d k^{2}}-\frac{s^{2}}{\left(1+s^{2} k^{2}\right)^{2}}+\frac{\epsilon}{2} \cos (k+\lambda), \\
\chi(k, \lambda)=\sqrt{1+s^{2} k^{2}} \int d x e^{-i k x} F(x) .
\end{gathered}
$$

Eq. (A3) is readily solved. It's eigenfunctions $\chi_{p}(k, \lambda)$ are defined by

$$
\mathcal{H} \chi_{p}(k, \lambda)=-\mu_{p}(\lambda) \chi_{p}(k, \lambda)
$$

with corresponding eigenvalues $\mu_{p}(\lambda)$. These eigenfunctions form a complete set in the $k$ space. Back substitution into Eqs. (A5) and (A2) yields the eigenvalues and eigenfunctions 
for the 2D system Eq. (A1):

$$
\Psi_{p, \lambda}(x, \theta)=\sum_{l} e^{-i l(\theta+\lambda)} \int d k e^{i k(x-1)} \frac{\chi_{p}(k, \lambda)}{\sqrt{1+s^{2} k^{2}}},
$$

where $\lambda$ has been written as a subscript to indicate the fact that $\lambda$ is now the second good 'quantum' number of the system Eq. (A1). We can show that all the eigenfunctions of Eq. (A1) are given by Eq. (A7), by showing that $\Psi_{p, \lambda}(x, \theta)$, with $p$ labeling the complete set $\chi_{p}(k, \lambda)$ and $\lambda$ running from 0 to $2 \pi$, is complete in the 2D space $(x, \theta)$. Thus, any function $\Phi(x, \theta)$ can be written as a superposition of $\Psi_{p, \lambda}$ :

$$
\begin{aligned}
\Phi(x, \theta) & =\sum_{p} \oint d \lambda C_{p}(\lambda) \Psi_{p, \lambda}(x, \theta) \\
& =\sum_{1} e^{-i l \theta} \int d k \oint d \lambda e^{i k(x-1)-i \lambda \lambda} \frac{\sum_{p} C_{p}(\lambda) \chi_{p}(k, \lambda)}{\sqrt{1+s^{2} k^{2}}} .
\end{aligned}
$$

Comparing to Eq. (2), we find that Eq. (A8) is equivalent to Eq. (7). Thus the procedures outlined in section (II) is nothing nore than the expansion in a new set of base functions $\Psi_{p, \lambda}$, which, in the case of the approximate translational invariance, is more convenient than the usual Fourier basis. 


\section{Appendix B: Coupling Matrix Elements}

From the orthogonality properties, we have

$$
\left\langle\chi_{0}|\mathcal{L}[\lambda]| \psi_{\tilde{g}}^{( \pm)}\right\rangle=-\frac{1+\epsilon \tilde{g}}{4}\left\langle\chi_{0} \mid \psi_{\tilde{g}}^{( \pm)}\right\rangle=0 .
$$

Differentiating Eq. (Bl) with respect to $\lambda$, we get

$$
\left\langle\chi_{0} \mid \frac{\partial \psi_{\tilde{g}}^{( \pm)}}{\partial \lambda}\right\rangle=\frac{2}{\tilde{g}-g_{0}(\lambda)}\left\langle\chi_{0}|\sin (k+\lambda)| \psi_{\tilde{g}}^{( \pm)}\right\rangle .
$$

Straight forward algebra yields

$$
\begin{aligned}
\left\langle\chi_{0} \mid \frac{\partial \psi_{\tilde{g}}^{( \pm)}}{\partial \lambda}\right\rangle= & \frac{4 i}{\epsilon\left(\tilde{g}-g_{0}\right)} \frac{2 \beta}{N_{c} N_{0}\left(\tilde{g}^{2}-g_{0}^{2}\right)} \times \\
& \left\{\left(\tau_{r} \tau_{R}+1\right) e^{i \theta / 2} \operatorname{Re}\left[e^{i\left(\theta-\theta_{+}\right) / 2}\left(1-B_{+}+\frac{2 i \beta_{0}}{\beta-\tilde{g}-Y_{+}}\right)\right]\right. \\
& \left. \pm\left(\tau_{r}-\tau_{R}\right) e^{i \theta / 2} \operatorname{Re}\left[e^{i\left(\theta-\theta_{-}\right) / 2}\left(1-B_{-}+\frac{2 i \beta_{0}}{\beta-\tilde{g}-Y_{-}}\right)\right]\right\} .
\end{aligned}
$$




\section{References}

1. C.Z. Cheng, L. Chen, and M.S. Chance, Annals of Physics (N.Y.) 161, 21 (1985).

2. C.Z. Cheng and M.S. Chance, Phys. Fluids 29, 3695 (1986).

3. S. Riyopoulos and S.M. Mahajan, Phys. Fluids 29, 731 (1986).

4. L. Chen, in Theory of Fusion Plasma, edited by J. Vaclavik, F. Troyon, and E. Sindoni (Centre de :cherche Paul Pascal, Talence, 1988), p. 327.

5. G.Y. Fu and J.W. Van Dam, Phys. Fluids B 1,1949 (1989).

6. G.Y. Fu and C.Z. Cheng, Phys. Fluids B 2, 985 (1990).

7. K.L. Wong, R.J. Fonck, S.F. Paul, D.R. Roberts, E.D. Fredrickson, R. Nazikian, H.K. Park, M. Bell, N.L. Bretz, R. Budny, S. Cohen, G.W. Hammett, F.C. Jobes, D.M. Meade, S.S. Medley, D. Mueller, Y. Nagayama, D.K. Owens, and E.J. Synakowski, Phys. Rev. Lett. 66, 1874 (1991).

8. W.W. Heidbrink, E.J. Strait, E. Doyle, and R. Snider, Nucl. Fusion 31, 1635 (1991).

9. F. Zonca and L. Chen, Phys. Rev. Lett. 68, 592 (1992).

10. M.N. Rosenbluth, H.L. Berk, J.W. Van Dam, and D.M. Lindberg, Phys. Rev. Lett. 68, $596(1992)$.

11. M. N. Rosenbluth, H. L. Berk, J. W. Van Dam, and D. M. Lindberg, Phys. Fluids B 4, 2189 (1992).

12. H.L. Berk, J.W. Van Dam, Z. Guo, and D.M. Lindberg, Phys. Fluids B 4, 1806 (1992). 
13. N.N. Gorelenkov and S.E. Sharapov, Phys. Scr. 45, 163 (1992).

14. R.R. Mett and S.M. Mahajan, Phys. Fluids B 4, 2885 (1992).

15. H. Ye, Z. Sedlacek, and S.M. Mahajan, to be published.

16. X.D. Zhang, Y.Z. Zhang, and S.M. Mahajan, Phys. Fluids B 5, 1257 (1993).

17. Y.Z. Zhang and S.M. Mahajan, Phys. Lett. A 157, 133 (1991).

18. Y.Z. Zhang, S.M. Mahajan and X.D. Zhang, Phys. Fluids B 4, 2729 (1992).

19. J.W. Connor, R.J. Hastie and J.B. Taylor, Proc. R. Soc. A 365, 1 (1979).

20. Y.C. Lee and J.W. Van Dam, in Proc. Finite Beta Theory Workshop, Varenna Summer School of Plasma Physics, eds. B. Coppi and B. Sadowski (U.S. Department of Energy, Office of Fusion Energy, CONF-7709167 (1979).

21. A. Glasser, ifid. p. 55.

22. R. Dewar, Theory of Fusion Plasma, Proceedings of the workshop held at Villa CipressiVarenna, Italy Aug. 24-28, 1987. ed. by A.Bondeson, E. Sindoni, and F. Troyon (Editrice Compositori Societa Italiana di Fisica, Bologna Bo Italy, 1988), p. 107.

23. R. Dewar and Y.Z. Zhang, Bull. Am. Phys. Soc. (1992). 


\begin{tabular}{||c|c|c||}
\hline$s$ & $\delta_{1}$ & $\delta_{2}$ \\
\hline 0.5 & -0.2151 & -0.7100 \\
\hline 0.8 & -0.1938 & -1.1570 \\
\hline 1.0 & -0.1761 & -1.3013 \\
\hline 1.5 & -0.1400 & -1.4515 \\
\hline 2.0 & -0.1152 & -1.5042 \\
\hline
\end{tabular}

Table I. Phase shifts $\left(\delta_{1}\right.$ and $\left.\delta_{2}\right)$ for various shear parameters $s$. 


\section{Figure Captions}

1. Damping rate as a function of $m \hat{\epsilon}$ for various shear parameters $s$. (a): $s=0.5$, (b): $s=1.0,(\mathrm{c}): s=1.5,(\mathrm{~d}): s=2.0$.

2. Comparison of the present calculation (thin line) with the results of Ref. 11 (thick line). 


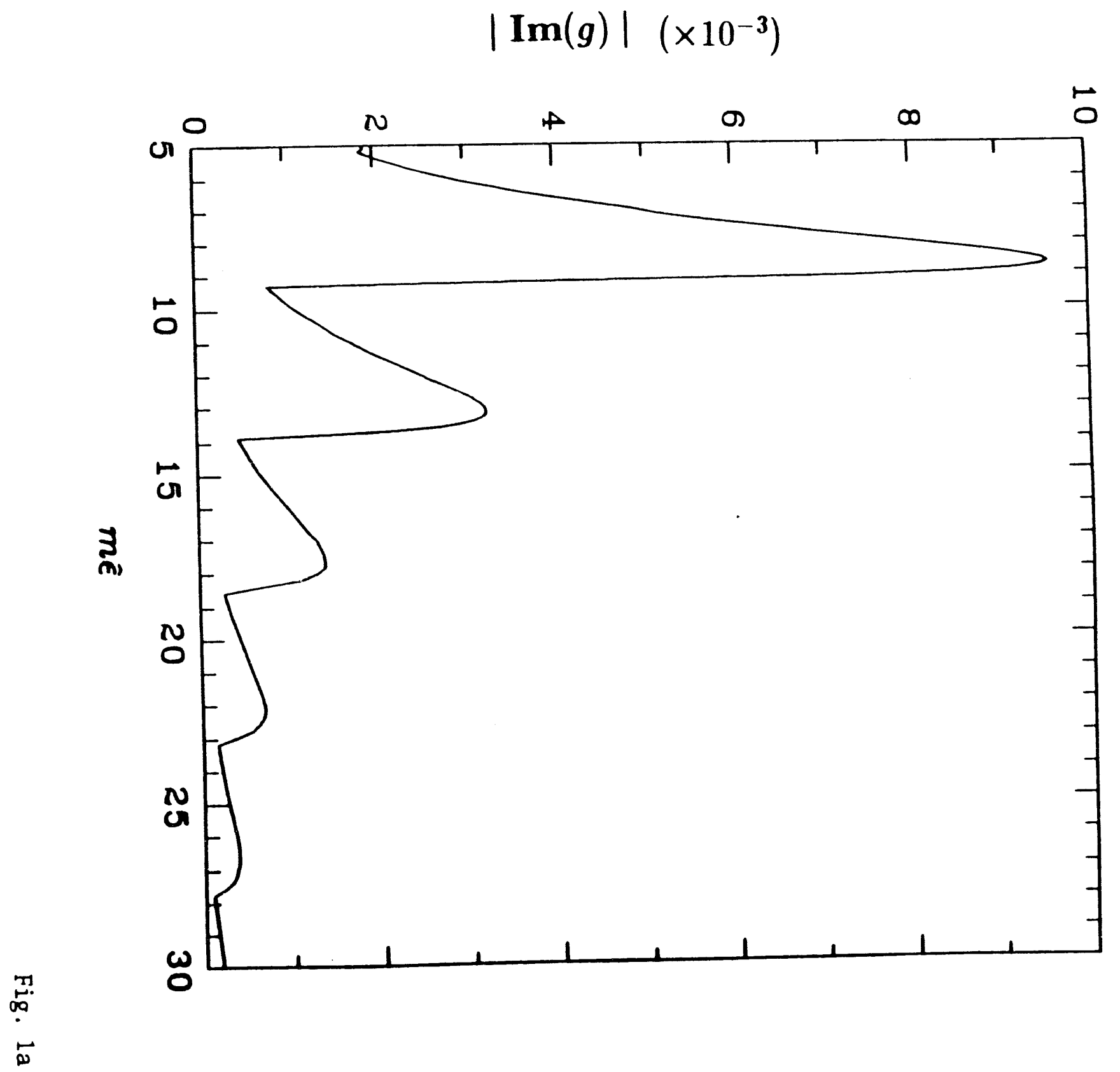




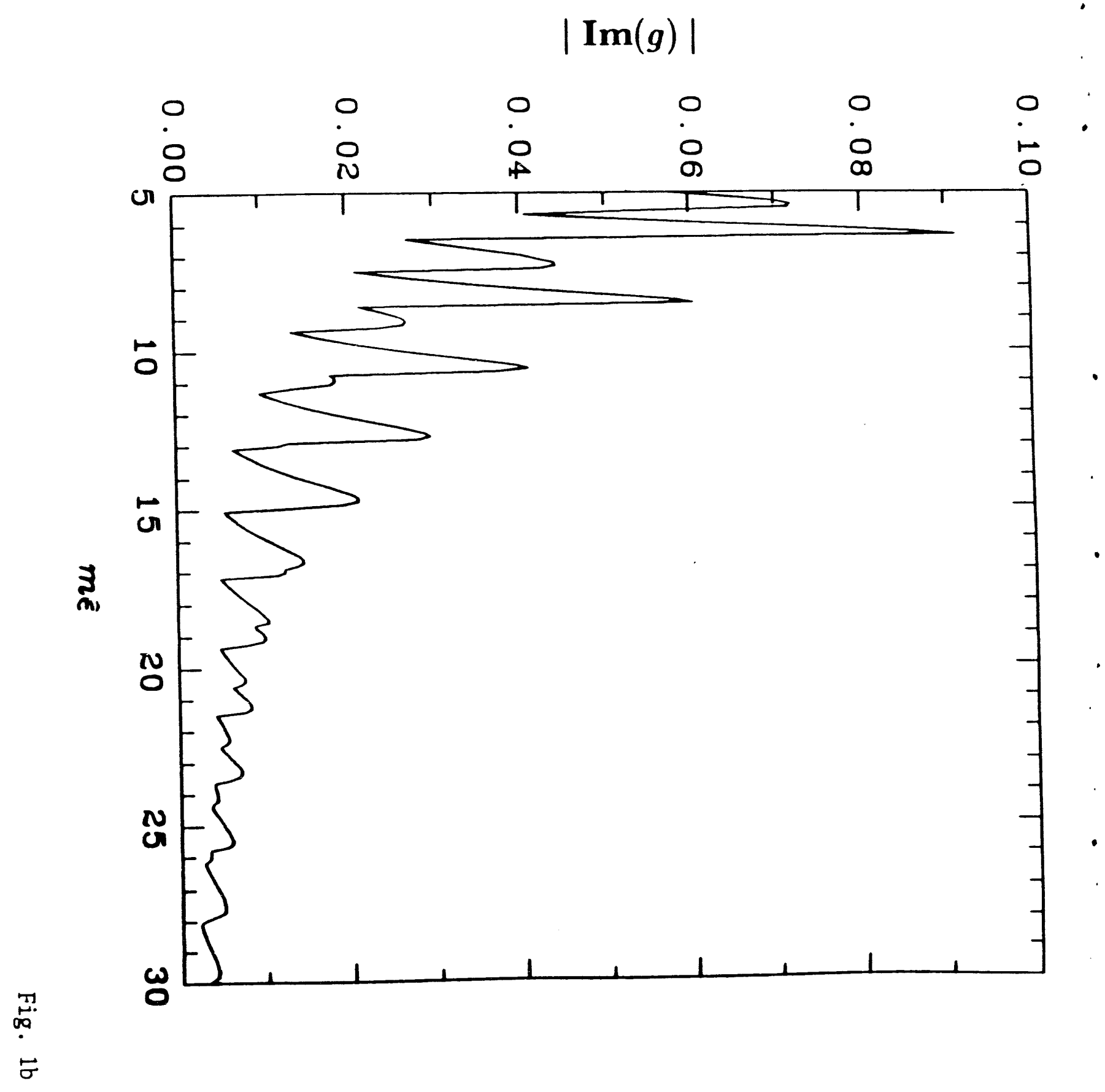




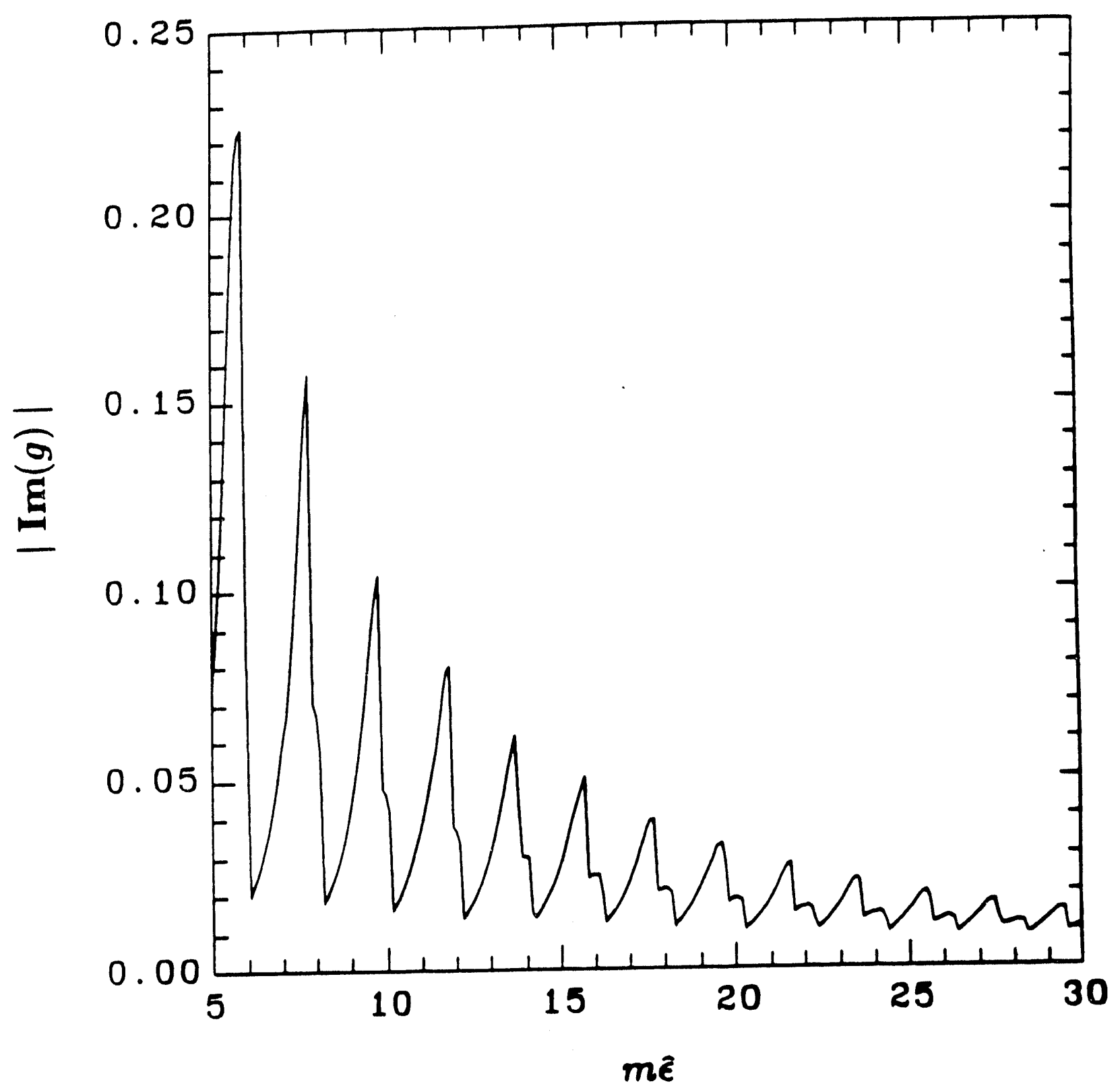

Fig. 1c 


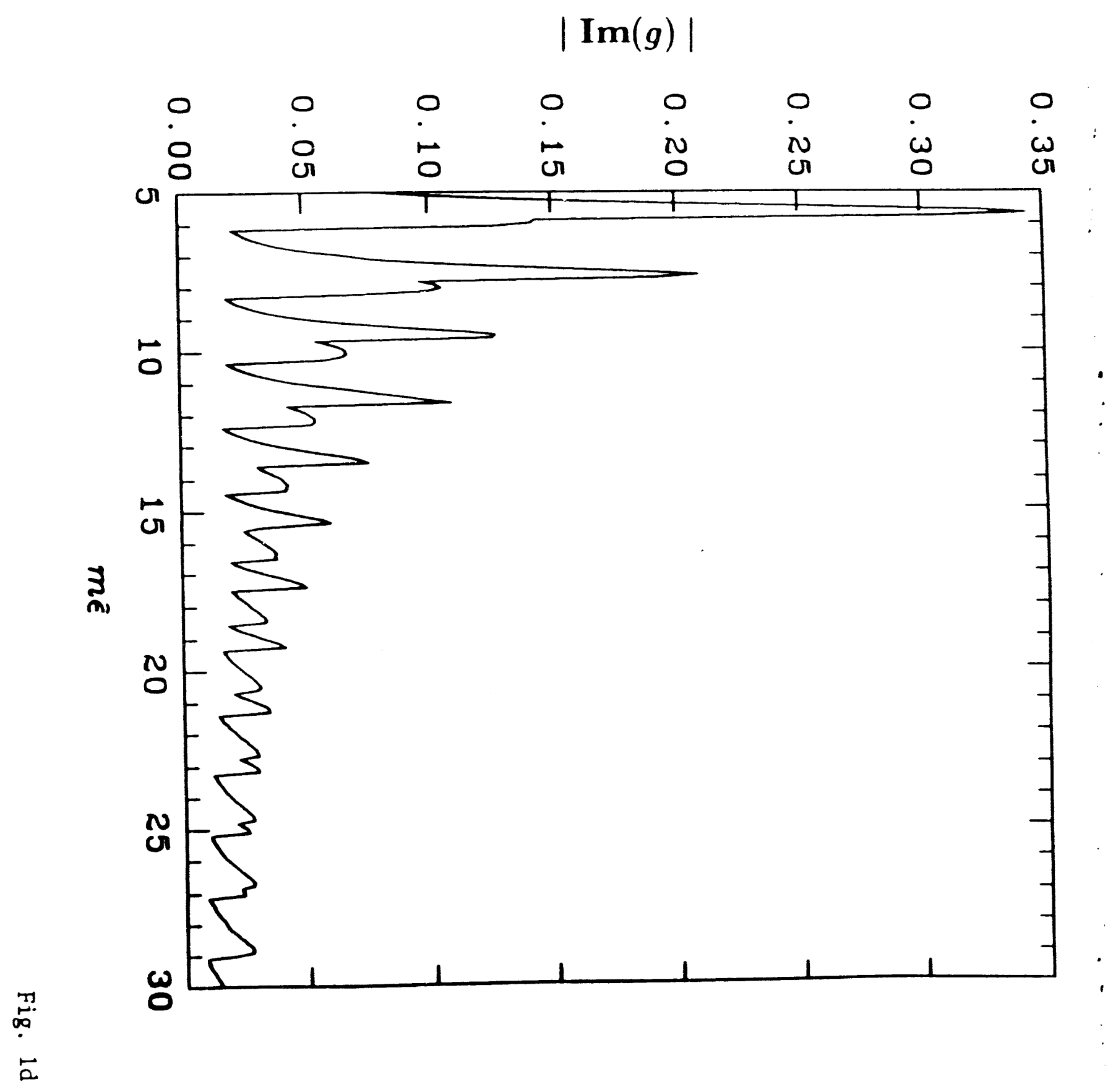




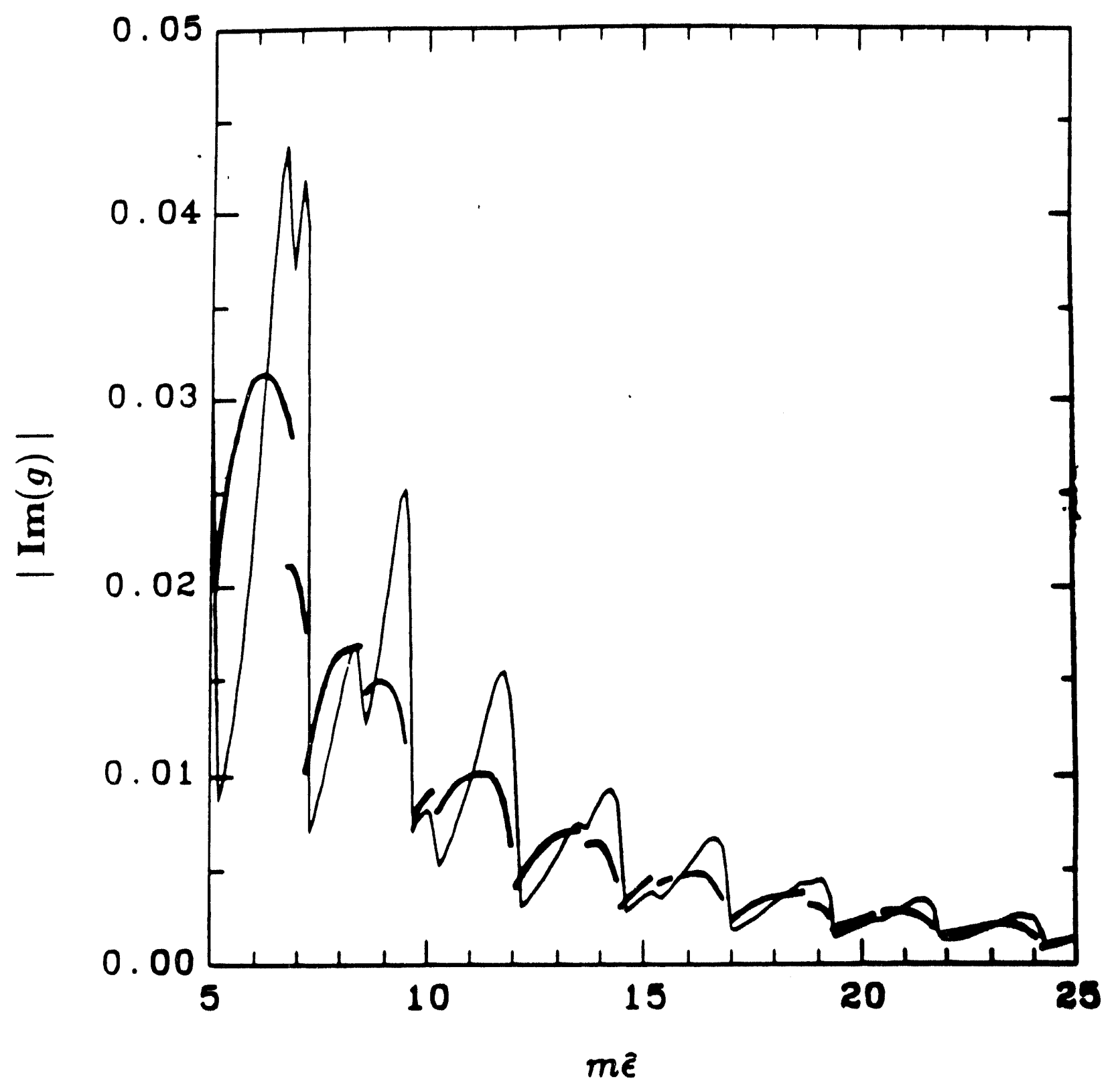

Fig. 2 

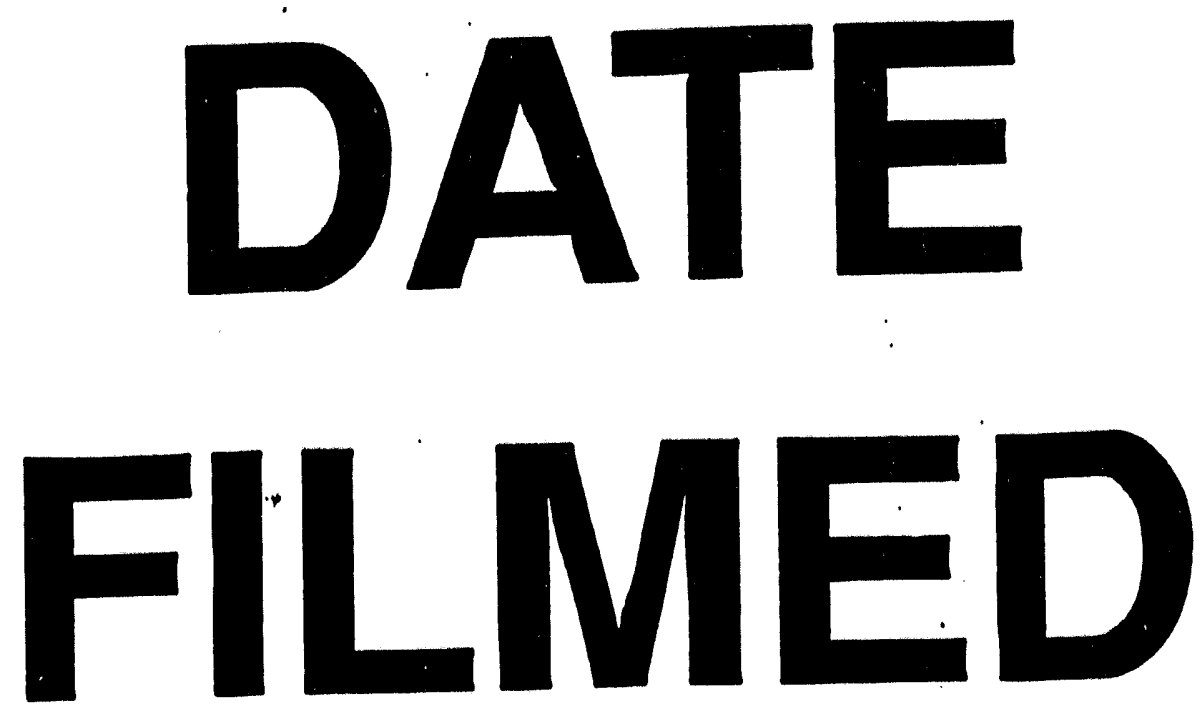

$12 / 6 / 93$
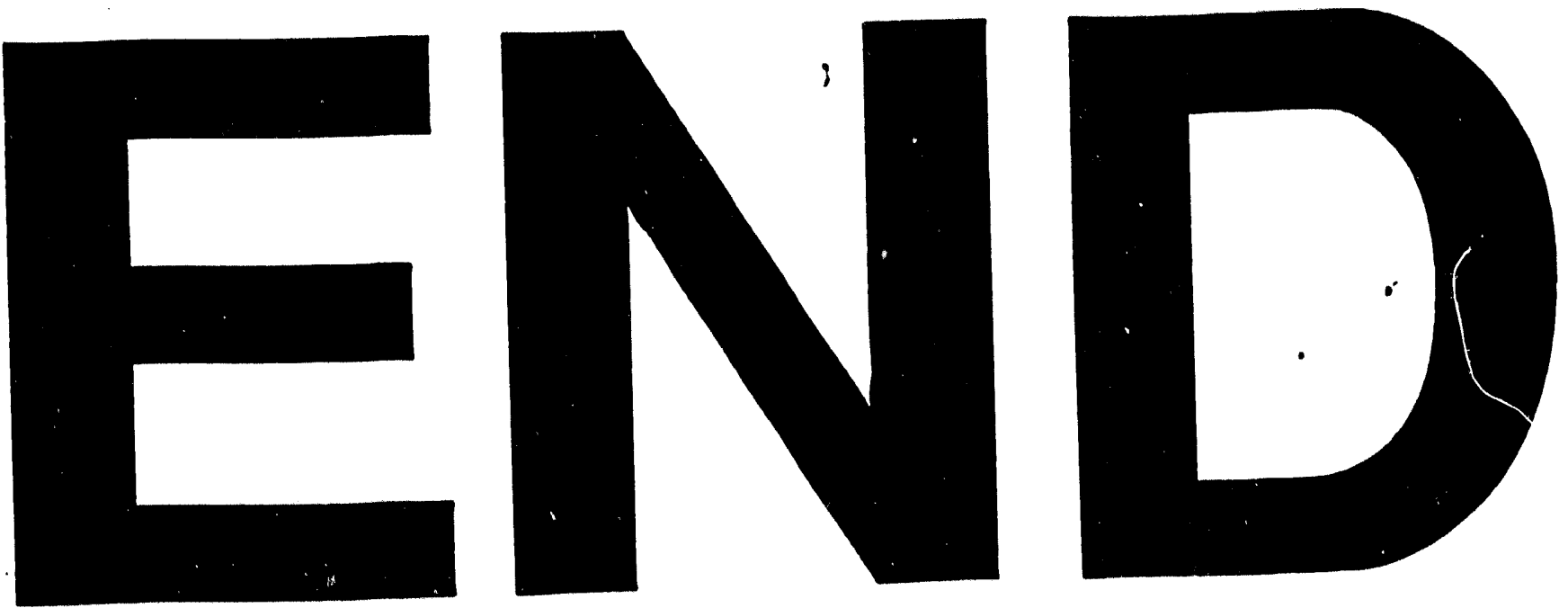


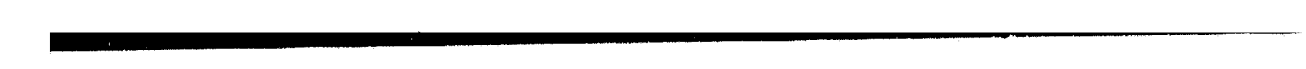

